Portland State University

PDXScholar

Engineering and Technology Management

Faculty Publications and Presentations

$10-8-2018$

\title{
Novel Approach to Managing Technological Entrepreneurship Using a Model-Based Systems Approach to Develop Low Cost Earth Orbiting Satellites
}

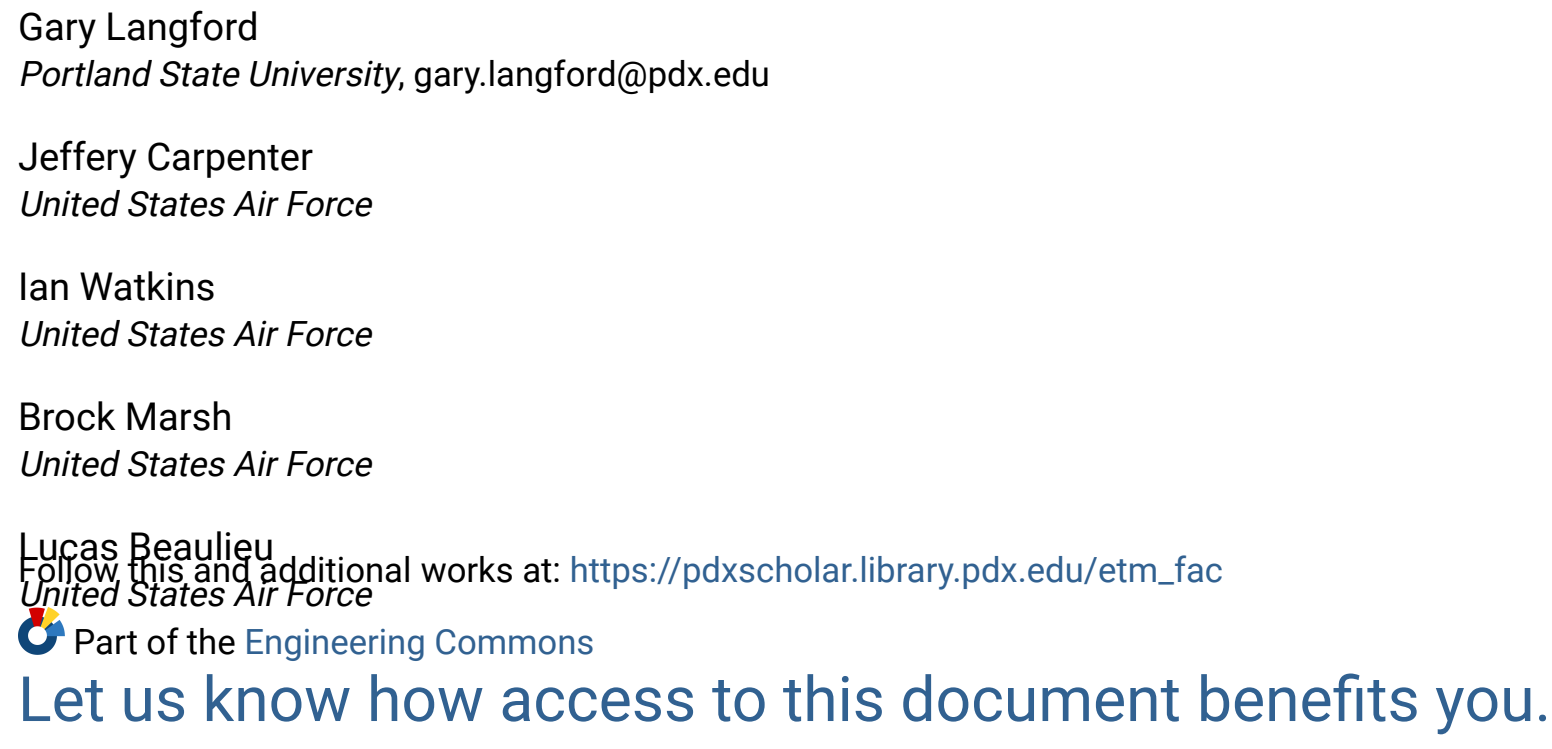

\section{Citation Details}

G. Langford, J. Carpenter, I. Watkins, B. Marsh and L. Beaulieu, "Novel Approach to Managing Technological Entrepreneurship using a Model-Based Systems Approach to Develop Low Cost Earth Orbiting Satellites," 2018 Portland International Conference on Management of Engineering and Technology (PICMET), Honolulu, HI, 2018, pp. 1-6.

This Article is brought to you for free and open access. It has been accepted for inclusion in Engineering and Technology Management Faculty Publications and Presentations by an authorized administrator of PDXScholar. Please contact us if we can make this document more accessible: pdxscholar@pdx.edu. 


\title{
Novel Approach to Managing Technological Entrepreneurship Using a Model-Based Systems Approach to Develop Low Cost Earth Orbiting Satellites
}

\author{
Gary Langford ${ }^{1}$, Jeffery Carpenter ${ }^{2}$, Ian Watkins ${ }^{3}$, Brock Marsh ${ }^{4}$, Lucas Beaulieu ${ }^{5}$ \\ ${ }^{1}$ Engineering \& Technology Management, Portland State University, Portland, Oregon, USA \\ ${ }^{2}$ United States Air Force (Ret), USA \\ ${ }^{3}$ United States Air Force, $9^{\text {th }}$ Bomb Squadron, Dyess AFB, Abilene TX, USA \\ ${ }^{4}$ United States Air Force, USA \\ ${ }^{5}$ United States Air Force, 27th Special Operations Aircraft Maintenance Squadron, Cannon AFB, NM, USA
}

\begin{abstract}
The ability to send low cost satellites into space has changed the satellite industry and vastly opened up the use of satellites to transform data into information for individuals, organizations, commercial companies, and governments. This information can be corroborated with other sources of data to evaluate the availability of precious resources, e.g., potable water, agriculture; to forecast upcoming famines or diseases; and to perform mapping, communications, and competitive analyses. The cost of owning a satellite is less than \$100,000 (using commercial parts) to $\$ 1,000$ (built by school kids). Launch costs are tens of thousands of dollars, which can be eliminated through subsidies. Compare these costs to $\mathbf{\$ 2 0 0}$ million to $\$ 1$ billion costs for similar functionality, higher performance and greater durability-an interesting trade space that favors multiple low cost flights versus significantly higher costs for permanent satellites for an increasing number of applications. The challenge is to form and manage a development team of unskilled professionals, high school students, or university students to meet deadlines and flight-standards. These team members are likely highly motivated and unskilled. This paper describes the technical management strategy and techniques used to develop the $10 \mathrm{~cm}$, 6-sided CubeSats.
\end{abstract}

\section{INTRODUCTION}

Technical management is impacted heavily by countless systems, steeped in a swamp of systems of systems. Truth be told, we are immersed in systems that dramatically impact our decisions in ways we often do not appreciate or understand. Systems interact with our efforts in social, economic, political, and situational domains. Sometimes, unintended consequences of those interactions cause technical managers to exceed their budgets, lose access to required resources, fail to meet schedule demands, and deliver less functional performance and quality than planned. The recognition that systems rule our lives suggests a systems approach is essential for technical management. The technical manager is responsible for achieving enduring outcomes given unforeseeable long-term market forecasts, mercurial mechanisms that drive customer and user behaviors, and unanticipated reactions from competitors. As daunting as those capricious factors are, the technical project manager for space project takes on additional responsibilities when merging technical innovations into the myriad of systems that will portend success in three very different operational environments - Earth (office, lab, industrial facilities, ruggedized for long-term exposure outdoors); rocket launch (acceleration, vibration, and shock); Earth orbit (thermosphere/Kármán line); and solar system (protons, cosmic radiation, ultra-violet radiation, and x-rays).

The purpose of this paper is to describe a model-based systems approach to technical management that emphasizes teamwork to design, build, and operate CubeSats (see figure 1 below) in the systems and systems of systems of Earth, rocket, thermosphere, and solar system. While the systems approach to technical management is not new, a model-based systems approach is novel and without precedence.

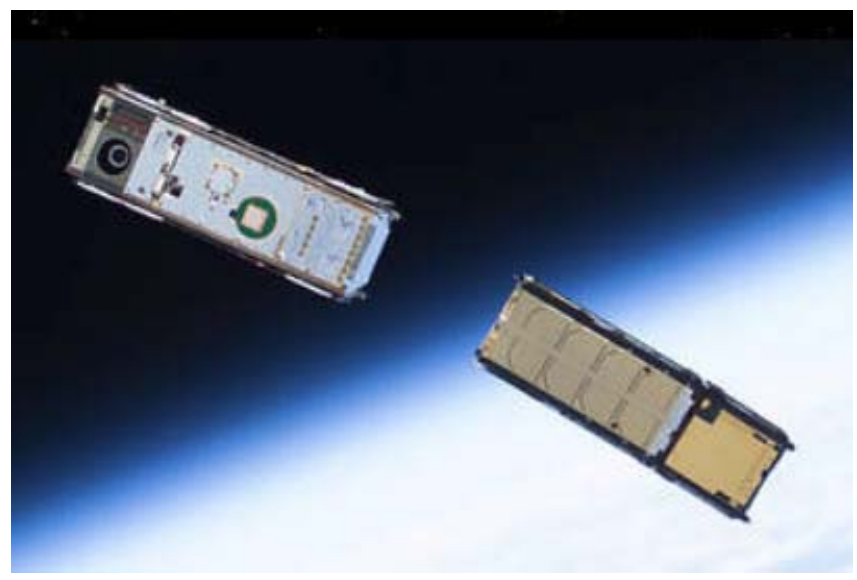

Fig. 1. NASA CubeSat

This paper focuses on how to develop the necessary ability for highly skilled graduate students who are senior professionals in the United States Air Force to become technical managers and teammates on a complex satellite project that spans all four development and operational 
domains. These students, unfamiliar with designing, building, testing, and operating space hardware and software, will mature their leadership capacity by discovering and employing leadership behaviors to form and build the teamness demanded by the students' will to succeed.

\section{BASIS OF TECHNICAL MANAGEMENT}

We can think of the objective of project management as the administration of work of a group of people and their instruments of labor to produce a desired outcome. The typical examples of technical management of a project (including computer systems, programming, and networks are a very small portion of building, launching, and operating satellites). And, not only are these satellite environments severe and inhospitable, they also render the space hardware inaccessible to service calls, maintenance, and replacements. Technical management builds on extrapolations and extensions of technology into innovations that are substantially proven by laboratory results, modeling and simulation, and prototypes. Yet developing new products and services premised on immature technology will challenge the historically most successful development teams. Technical managers must reconcile technology realism with that of innovative prowess and plan for product introductions in increments or phased releases. Technical management needs to be embedded in the systems in which organizations thrive and projects flourish.

\section{TeChnOlogy Management ReQuiRes EMBEDDEDNESS}

That the very foundation of technical management should be built on the realization that technology embeddedness leads to organizational change was suggested in 2007 by Volkoff, Strong, and Elmes [1]. When organizational elements are embedded in technology, the processes derived from interactions between the project stakeholders and those stakeholders and the physical objects within the systems boundaries of the project, the associated emergent take on tangible substance and physical meaning within the context of systems. This material aspect and their logical behavioral manifestations were recognized by Feldman and Pentland [2]. Importantly, Feldman and Pentland identified a means whereby the functions (suitably referred to as roles) carried out by individuals embed their interactions with others within the contexts of the systems and systems of systems of their organizations and projects. These interactions are extensible to stakeholders and their respective organizations. The actions resulting from this technology embeddedness can be described as a mechanism that is comprised of processes and activities that assume legitimacy in action and fact by leadership brazened by role, presence, behavior, and success. This mechanism provides a new perspective of technical management - one that reinforces behavior with the subtle threat of truculence as essential to organization and project success.

\section{SEVEn Essential Behaviors of TeChNiCAL MANAGEMENT}

While multiple behaviors are deemed essential to technical management, at least one team member in a position of responsibility and authority must have the essential seven "behaviors". The seven essential behaviors are (1) be forthright in speaking your mind without fear of disagreeing or retribution, i.e., treat people as friends - everyone is a contributing member of the system; (2) consider the facts, the alternatives, and the requirements before taking what others might consider to be risks that maximize worth and minimize losses to meet objectives, i.e., there is no risk, only different alternatives to accomplishing your just purpose - advantage use of resiliency built into systems processes, redundancies, and shadow operations; (3) be personable and direct in all communications - stay above politics, i.e., pragmatism guards against nonrealistic decisions which reduces losses within the system; (4) stay focused on being effective to achieve goal, objective, and appropriate outcomes - actions on task minimize non-productive system activities; (5) use principles to develop rules of thumb and rules of dumb from which to determine measures of effectiveness - processes built on systems principles facilitate appropriate decisions to rapidly supply needed energy, matter, material wealth, and information; (6) champion, safeguard, and steward the team - the system supports its constituents and the constituents support the system; and (7) delegate to facilitate ownership of ideas, decisions, and innovation - recognizes the advantages and constraints of systems of systems and systems.

\section{CONTEXT FOR TECHNiCAL MANAGEMENT}

\section{A. Technical Management of Space Products' Complexities}

Managing technology development should not done in isolation from the very contexts in which the innovation must eventually find its uses. While managers may talk of company sales and profits, and users and customers, the influences of systems outside their organizations have dominant control on determining customer purchases and users' acceptance. Examples of these influences are company's perception of their brand versus interpretations on social media, pricing to competition rather than on the basis of cost, and worth of product functionality and functional performances. For spacebound products, functional longevity, number of modes for degraded operations, effectiveness of on-orbit status and checkout, anomaly resolution timeframe are a few measures by which customers determine value and complexity. Customer involvement with a space product means commitment of funds and resources to support that space product for at least a portion of the product lifecycle.

\section{B. Technical Management and Systems Engineering-One in the Same Context}

On occasion, we interact with a system whose influence and complexities are underestimated or underappreciated by the technology manager. For instance, when operating a $1 \mathrm{~kg}$ satellite (CubeSat) in outer space, the development team must design and test for interactions with objects both within our solar system, e.g., planet Earth's gravitational anomalies, the sun's radiation, meteor belts, and human-made space junk; as well as interstellar radiation and objects that track into our solar system. In its orbit $160 \mathrm{~km}$ above the Earth's surface, CubeSats can also be harmed as well as cause damage to other operating 
satellites and space probes. It is disastrous to ignore or misinterpret the interactions between the CubeSat system of systems and the countless physical entities that comprise our solar system. The conceptual design work needs to reflect a set of acceptable requirements that are consistent with principlebased best practices. Systems engineering is the accepted means of developing space products in a rigorous, methodical fashion by NASA [3] and the U.S. Department of Defense [45]. In other words, systems engineering and technical management are one in the same, but different in their focus. Technical management views the same information seen by the systems engineer from a budget and schedule perspective premised on delivering the required technical performances. The systems engineer views the same information seen by the technical manager from the technical performances that are required within a negotiated schedule - premised on delivering with budget. The rationale for that integration of technical management and systems engineering is to "right the culture".

\section{PRIMER For TeChNiCAl MANAGEMENT OF COMPLEXITY}

The processes for developing technology products are the same as for managing the development of technology products [6]. Within the constructs of systems engineering, technical management is both proactive and reactive with regards to determining when a task has completed its exit criteria, when the next task shall begin, and how to assess the critical path (that favors a 3-day +2 regimen within a functional work breakdown structure). This regimen has a 3-day minimum delegated work activity with 1 day to assess and evaluate inputs to the task and 1 day to summarize and handoff outputs of the task. Micromanagement occurs only in the 2 day during inputs and outputs to the task. Overall technical management is focuses on the weekly "burn rate" (labor and contract expenditures on an accrual basis) with data prepared by forecasting earned value rates per development stage according to a systems engineering process model and level of effort accounted on a weekly basis. Often, risk assessment and evaluation is part of the technical management strategy.

\section{THE FundAMENTALS OF SySTEMS AND SYSTEMS OF SYSTEMS}

\section{A. Systems}

The distinction between notasystems, systems, and systems of systems is determined by whether certain conditions are fulfilled. A system is a bounded, dynamically stable group of objects and processes exhibiting intrinsic emergent properties derived from the interactions of energy, matter, material wealth, and information (EMMI) [7] which satisfies four conditions [8]. A system is defined as a group of interacting objects that are adaptively stable and reveal irreversible (natural) or nonreciprocal (artifactual) emergence. Interacting objects (in bounded aggregation) that satisfy four conditions are defined to be a system.

- The condition of Metastability-objects within the boundaries can change from one state of being dynamically stable to another state of being dynamically stable, where the conditions to be in one state are different than the conditions required to return to a previous state. Dynamic stability means to be resistant to change from a given state.

- The condition of Internal agility-objects within boundaries have agility and control to exchange EMMI in response to stimuli;

- The condition of External adaptability-objects within the boundaries adapt to changes in their external environment and context; and

- The condition of Nonreciprocal or irreversible emergence-objects within the boundaries are sensitive to changes in uses of EMMI.

Systems balance stability and flexibility through adaptive processes that serve to reoptimize the states of constituent objects through MIEN conditions. MIEN conditions are necessary and sufficient for a bounded aggregation of objects to be a system.

A space product that satisfies these four conditions is a system. By definition, all system elements must be integral to the operations of the system. Artifactual systems can be designed to extend essential system functionality and needed performance(s) at the expense of other needs of the system. In this manner, the artifactual system requires the use of EMMI to sustain operations. Space-based CubeSats require a source of electricity to power computers, communication equipment, and an array of sensors. The design and architecture of the network of CubeSats will a primary emphasis during the early development phase for the student team.

\section{B. Systems of Systems}

A system of systems is an integrated, interoperable set of systems that achieve a set of meta-system functions in which all constituent systems participate to varying degrees. Unlike systems, the integration of systems into an artifactual system of systems should not irreparably degrade or harm the constituent systems. That does not mean, however, that the constituent systems will not incur a loss as a consequence of joining, participating in, and perhaps leaving the system of systems. Constituent systems do lose EMMI. For every action, there is a loss of EMMI [7]. Each constituent system must retain its systemic behaviors and the system of systems must also maintain its systemic behavior. Therefore, each constituent system and the system of systems must individually sustain their four essential properties. However, the single characteristic element of the system of systems is found in the lifecycle of the constituent systems - do no harm to the constituent systems [8].

A system of systems is defined as a group of interacting objects (systems) that independently are adaptively stable and individually reveal irreversible (for natural systems) or nonreciprocal (for artifactual systems) emergence. Interacting objects (as a system) must either satisfy the four conditions as defined to be and remain a system, or rely on the actions of the constituent systems that comprise the systems of systems to satisfy certain conditions for the protasystem (a system that is life-lined to be satisfy its four conditions to demonstrate its systemness during the its' participation in the system of systems. A system of systems may sustain a protasystem so that it can be removed from the system of systems when the 
constituent systems depart. It takes a minimum of two constituent systems to be a system of systems.

- The condition of Metastability-objects within the boundaries of the protasystem may want or need to retain and use their ability to change from one state of being dynamically stable to another state of being dynamically stable, where the conditions to be in one state are different than the conditions required to return to a previous state. Dynamic stability means to be resistant to change from a given state.

- The condition of Internal agility-objects within the boundaries of the protasystem may want or need to have agility and control to exchange Energy, Matter, Material wealth, and Information (EMMI) in response to stimuli;

- The condition of External adaptability-objects within the boundaries of the protasystem may want or need to adapt to changes in their external environment and context; and

- The condition of Nonreciprocal or irreversible emergence - objects within the boundaries of the protasystem may want or need to be sensitive to changes in uses of EMMI. A space product that satisfies these four conditions is a system of systems.

\section{THE SYSTEMS APPROACH}

This paper introduces a reflexive model-based systems approach to determine emergent behaviors in complex systems and systems of systems. The goal of technical management is to develop and build to requirements that capture all the emergents in a system or systems of systems. It is the objective to formulate a set of relations within the confines of a causal domain to answer questions, solve problems, resolve conflicts, and to explore various concepts to enable a viable solution set for the CubeSat system of systems.

There are four paradigms of thought that need to be considered when formulating requirements for a system or system of systems. First, the requirements necessary and sufficient to capture the intended objectives for systemic activities, processes, emergence, and mechanisms need to be examined. Second, the problems that may arise from omitting requirements that encapsulate the systemic nature of systems or systems of systems need to delineated, objectified, assessed, evaluated, and characterized. Third, conflicts in requirements between processes of parts of constituent systems versus the activities of the system of systems (whole) must be reconciled with regards to design, architecture, and operational concepts. And fourth, deficiencies in requirements due to not building on systems theory, the model-based systems approach, the integrative ontology framework of objects and processes [8], and methods that may invalidate or limit the validity of the systems engineering work must be managed. Moreover, requirements for developing systems and systems of systems require validation through the systems perspective across all artifactual and natural mechanisms, i.e., enactments of processes and activities.

In brief, through the efforts of systems and systems of systems we build artifactual space products that are systems and systems of systems for operations in conjunction with the four systems and systems of systems that comprise the space products' contexts and domains of operation. In all cases, the requirements must include emergence, which requires multidisciplinary validation [7].

\section{TeChnical Management Using the Model-BASED SYSTEMS APPROACH}

Requirements are reflexive, i.e., self-referencing with knowledge about the self-reference. Alternatively said, the sole repository of context, attributes, design, and architecture are simultaneously the product and the requirements-the requirements are the surrogate of the product as is the product the surrogate of the requirements. Technical management must focus on the test for reflexivity to capture the nature and ideation of requirements. The co-referential relation between a reflexive set of requirements and its antecedent product is essential to establishing the congruence of model used for technical management and the exemplar used to manage, i.e., to plan, to communicate, or organize, to direct, to control, and to build teamness. The theory, framework, and approach used for technical management must also be reflexive with the model and exemplar [8].

Models of systems and systems of systems should be simplified agents of complexity. These agents should be imbued with sufficient fidelity to resemble the logic, structure, action, emergence, and interpretation of systems and systems of systems [7]. Based on a general theory of integration, a Leśniewski inspired interpretive integrative framework of part whole ontologies [7-8] and a reflexive model-based systems approach, the requirements for systems and systems of systems can be imbued with tractable mechanisms that enable causal actions, show emergence and context of interactions to move EMMI, incorporate boundary conditions to moderate (control) flows of EMMI by mechanisms (that can be either ontological or real), apply appropriate systemic processes and metrics to build capacities of EMMI and manage capacity utilizations, and prognosticate missing elements to provide meaningful lifecycle results. With the proper model and exemplar, technical management can capture all that is required to build a complex space product.

Therefore, the requisite mix and degree of "real" and "ideal" can be incorporated into the workings of technical management, such that mechanisms are logical, tractable, and graspable. All mechanisms show emergence because mechanisms are made up of interactions - all interactions result in emergence. However, while technical work must be relevant to the objectives for constructing the CubeSats, this paper does not address considerations that suggest or employ models planned for widespread use (in a commercial sense). Said alternatively, the technical management work requires considerable education at the graduate-level (Master's or Ph.D.). The team of graduate students is qualified to carry out technical management of the CubeSat development, test, and operational aspects. 
The objective is not to commercialize any particular systems approach, but rather to improve the efficaciousness in predicting emergence to improve requirements and technical management of same. Subsequent to the CubeSat project, the model-based systems approach can be reviewed and then possibly considered for wider use without the necessity for graduate education. Requirements to commercialize a model need to be imposed after the fidelity of the model-based systems approach is validated. Therefore, the strategy advocated here is to first establish a sound model-based systems approach using requirements that are validated using the best practices of systems engineering, and incorporating a sufficiency of systemness (essentially capturing all interactions and therefore revealing emergence).

\section{Description And Discussion Of CubeSat Design And OPERATIONS}

CubeSats are small, nano satellites built in blocks of cubic dimensions of $10 \mathrm{~cm}^{3}$ with a total mass of less than $1.33 \mathrm{~kg}$ per cube. In addition to being small they can also be combined with other CubeSats for increased capabilities. These capabilities can include weather monitoring and prediction, scientific experiments and measurements, data capturing and imaging, and even observation in our solar system and beyond. Even though they are small, they can include many parts, such as microprocessors, wiring, power supplies, wiring and cable, antennas, cameras, and data capturing devices and sensors. In order to get CubeSats into space, they can be launched independently or as part of NASA's CubeSat initiative which provides a free ride into space while secured inside a dispenser until the proper time to release. Regardless of the mission set for the CubeSat, the information it collects or uses must processed and used accordingly. The communications systems that relay this information and data primarily used telemetry and command systems which send and receive the information. This data, whether it is analog or digital must be relayed to a ground station and send to it via antenna onboard the CubeSat. It is conveyed to the ground after it is converted into a stream of binary numbers and sent to Earth. At the ground station, data is received and processed accordingly. There is also the ability to manipulate and alter many functions of the CubeSat remotely.

\section{CubeSat Development}

CubeSats developments can follow one of several paths of development. The NASA development path, is different from the Department of Defense development path, is different from the U.S. Air Force development path, is different from the U.S. Navy development path, is different from the development path used by students. The formalisms found in the government development efforts can be abbreviated with proper guidance. That guidance reduces the formalism of the documentation, yet maintains the rigor of the communications - essentially avoiding the formalisms that are associated with contract language requirements to satisfy acquisition policies. However, technical documentation is rigorous, individual notebooks are mandatory, and frequent meetings to share design and architecture progress are crucial. Most often, student projects incorporate an agile design and development philosophy, often building prototypes that are then modified to be used for destructive testing. The tenor of the team is to maintain details on configurations, keep the workspaces clean and orderly, and do not mix lunch with work. Learning can be accelerated by analyzing failure results. A detailed development handbook and glossary of terms is provided to each member of the student team. The student advisor has advised students developing space hardware with CubeSats and their predecessors since 1997.

\section{CONCLUSION}

CubeSats are distinguished by their capacity to carry out meaningful and valuable missions for a fraction of the price of the historically expensive spacecraft. An advantage for any spacecraft is to be part of a network topology of other data sensors, collectors, communicators, and processors. The evaluation of mission success is both by on-orbit performance as well as the work to design, develop, and test. Throughout the development and operational work, the general ontological framework, showing how components interact to provide the functional performances and models of behaviors, is used to plan, test, and integrate data flows and functioning throughout the CubeSat's lifecycle. Regardless of the type or duration of mission, the model of the various systems continues to lend insights into what will be next and then thereafter.

\section{DISCLAIMER}

Any views, opinions, findings, conclusions, or recommendations expressed or implied in this paper are those of the authors and do not reflect or represent the official policy or position of the United States Government, the United States Department of Defense, the United States Air Force, or the National Aeronautics and Space Administration, and nor do they reflect or represent the official policy or position of Portland State University, the Maseeh College of Engineering \& Computer Science, or the Engineering and Technology Management Department.

\section{REFERENCES}

[1] University of Texas description of Communication Subsytem, Available:http://www.ae.utexas.edu/courses/ase463q/design_pages/sprin g03/cubesat/web/Paper\%20Sections/4.0\%20Communication\%20Subsyst em.pdf

[2] NASA "How do CubeSats Get Into Orbit" Published Apr 14, 2017 : https://www.youtube.com/watch? $v=$ pnRdIyIWI0k\&feature=youtu.be

[3] NASA "What Does a Cubesat Do?" Published Apr 13, 2017: https://www.youtube.com/watch?v=BLJBVkLVGHE\&feature=youtu.be

[4] O. Volkoff, D. M. Strong, and M. B. Elmes, "Technological Embeddedness and Organizational Change," Organization Science, vol. 18, no.5, pp. 832-848, 2007.

[5] M. S. Feldman, and B. T. Pentland, "Reconceptualizing organizational routines as a source of flexibility and change," Admin. Sci. Quart., vol. 48, no. 1, pp. 94-118, 2003.

[6] NASA Systems Engineering Handbook, Rev. 2. 2017; Available: https://ntrs.nasa.gov/archive/nasa/casi.ntrs.nasa.gov/20170001761.pdf

[7] Office of the Deputy Under Secretary of Defense for Acquisition, Technology, Systems and Software Engineering, "Systems Engineering 
Guide for Systems of Systems," Version 1.0. Washington, DC: ODUSD(A\&T)SSE, 2008.

[8] Systems Engineering Primer \& Handbook. Space \& Missile Systems Center U.S. Air Force. 2004, January, 15. 2nd edition. Available: http://www.acqnotes.com/Attachments/SMC\%20System\%20Engineerin g\%20Handbook.pdf

[9] A. P. Sage and J.E. Armstrong, Introduction to Systems Engineering. New York, John Wiley \& Sons, Inc. 2000.
[10] G. O. Langford, Engineering Systems Integration: Theory, Metrics and Methods. Boca Raton, Florida, CRC Press/Taylor \& Francis. 2012.

[11] G. O. Langford. "System of Systems Process Model," in Engineering Emergence: A Modeling and Simulation Approach, L. Rainey and M. Jamshidi, Eds. Boca Raton, Florida: CRC Press/Taylor \& Francis. 2018 (in press). 